This item was submitted to Loughborough's Research Repository by the author.

Items in Figshare are protected by copyright, with all rights reserved, unless otherwise indicated.

\title{
QFT design for uncertain non-minimum phase and unstable plants revisited
}

PLEASE CITE THE PUBLISHED VERSION

PUBLISHER

(C) Taylor \& Francis

LICENCE

CC BY-NC-ND 4.0

REPOSITORY RECORD

Chen, Wen-Hua, and Donald J. Ballance. 2008. "QFT Design for Uncertain Non-minimum Phase and Unstable Plants Revisited”. figshare. https://hdl.handle.net/2134/3858. 
This item was submitted to Loughborough's Institutional Repository (https://dspace.lboro.ac.uk/) by the author and is made available under the following Creative Commons Licence conditions.

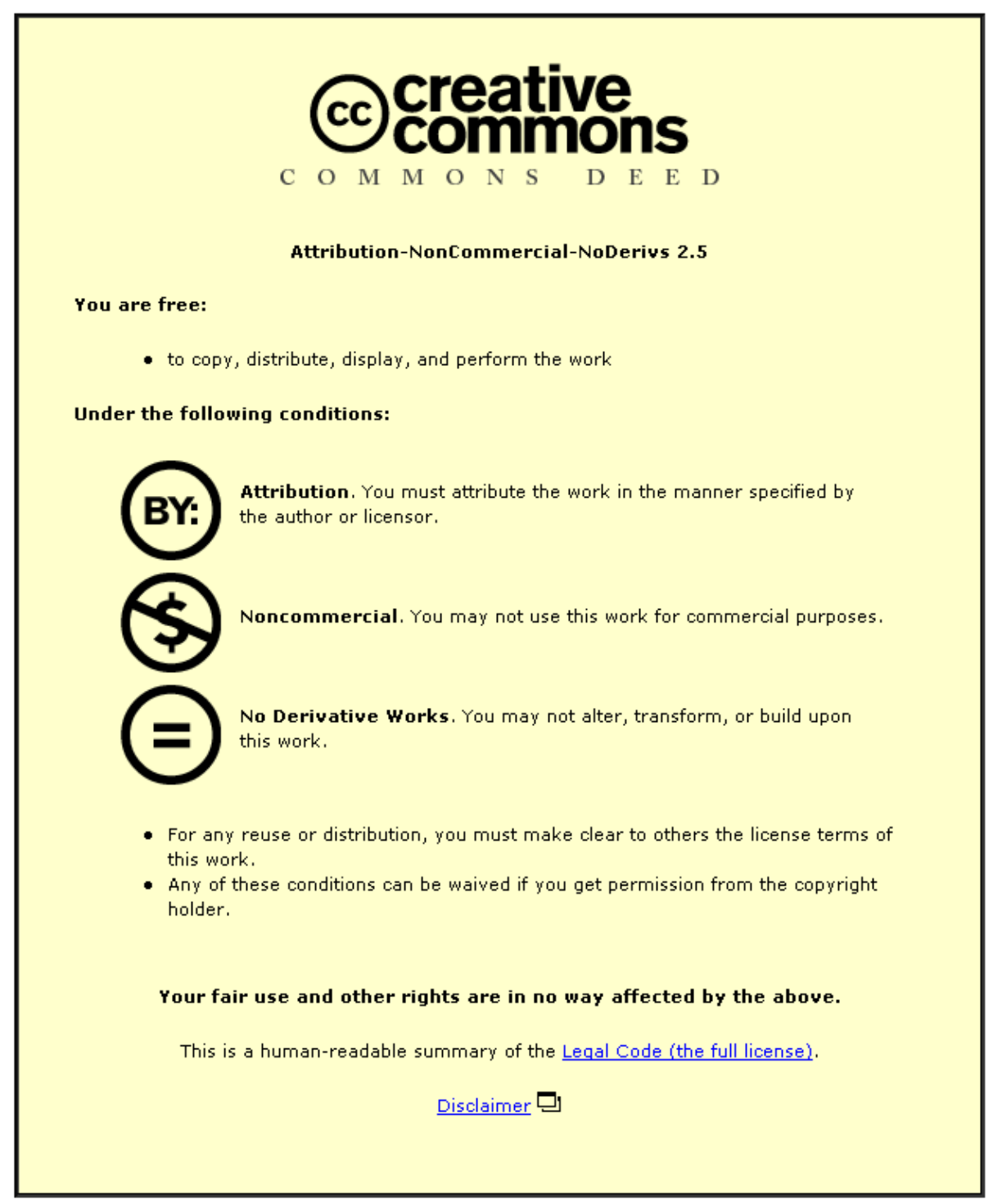

For the full text of this licence, please go to: http://creativecommons.org/licenses/by-nc-nd/2.5/ 


\title{
QFT Design for Uncertain Non-Minimum Phase and Unstable Plants Revisited
}

\author{
Wen-Hua Chen ${ }^{\dagger}$ and Donald J. Ballance ${ }^{\ddagger}$ \\ $\dagger$ Department of Aeronautical and Automotive Engineering \\ Loughborough University \\ Loughborough, Leicestershire LE11 3TU \\ $\ddagger$ Centre for Systems \& Control \\ and Department of Mechanical Engineering \\ University of Glasgow \\ Glasgow G12 8QQ, UK
}

Tel: 01413305178

Fax: 01413304343

\begin{abstract}
Design method for uncertain non-minimum phase and unstable plants in the Quantitative Feedback Theory (QFT) developed by Horowitz and Sidi (Horowitz and Sidi, 1978; Horowitz, 1992) is revisited in this paper. It is illustrated that the existing method may not work since some design rules have not been clearly specified by several examples including non-minimum phase plants and unstable plants. Then stability of a new nominal plant is carefully examined and analysed, and an improved design method is presented. The result in this paper provides mathematical justification of the QFT design procedure for non-minimum phase and unstable plants in Horowitz and Sidi (1978) and Horowitz (1992).
\end{abstract}

Keywords: Robust control, frequency domain, Quantitative Feedback Theory (QFT), non-minimum phase systems, unstable systems, robust stability 


\section{Introduction}

Quantitative Feedback Theory (QFT) was initially proposed by Horowitz and further developed by him and others (Horowitz, 1963; Horowitz and Sidi, 1972; Horowitz and Sidi, 1978; Horowitz, 1992; Houpis, 1995). It is considered as an efficient design method of robust controllers for plants with structured uncertainties, unstructured uncertainties and mixed uncertainties, and has been applied in aircraft, missiles, compact disk mechanisms, etc (Pachter and Houpis, 1997; Chait et al., 1994).

QFT is considered as an engineering oriented methodology. Unlike other robust control design methods, initially QFT was not received detailed mathematical analysis of its basis. Thus it has been criticised by lack of rigorous mathematical basis such as the existence of a controller; for example, see Doyle (1986), Ballance and Gawthrop (1992) and Ballance (1992). Although this situation has been significantly improved due to the efforts of many researchers (Yaniv and Horowitz, 1987; Nordgren et al., 1994; Jayasuriya and Zhao, 1994b; Jayasuriya and Zhao, 1994a; Chen and Ballance, 1999), there are still a number of "ad-hoc" design rules being used which are not explicitly evident in the stated design procedure.

This paper considers and adds to the design method for uncertain non-minimum phase and unstable plants proposed by Horowitz and Sidi (Horowitz and Sidi, 1978; Horowitz, 1992). This method was developed for uncertain non-minimum phase plants by Horowitz and Sidi (1978) about twenty years ago and then was extended to the unstable case by Horowitz in his book "Quantitative Feedback Theory" (Horowitz, 1992). This method is now recognised as an efficient method of dealing with non-minimum phase and unstable plants within the QFT formula; for example see Yaniv and Horowitz (1987) and Nordgren et al. (1994).

The key idea behind the Horowitz and Sidi method is to convert a loop-shaping problem for an unstable and/or non-minimum phase nominal plant to that for a stable minimum phase nominal plant by shifting robust stability and performance bounds. The reason is that in numerical design it is more convenient to work with a minimum phase function because the Bode integrals (Bode, 1945) can be used and the optimal loop shaping can be derived (Horowitz and Sidi, 1972; Horowitz and Sidi, 1978). In addition, although the same limitations imposed by right plane zeros and poles exist 
whatever choice is made and appear in one form or another, this method explicitly reveals the limitations on the open loop transmission $L(j \omega)$ and makes it much easier to see if assigned specifications can be satisfied. In what follows, this method is reviewed carefully and some mathematical basis is established. Several examples including the non-minimum phase case and the unstable case which show deficiencies in this method are given. That is, the stability requirement for the new nominal plant is not clearly specified. It is shown that in addition to shifting robust bounds, stability of the new nominal plant must be reconsidered. Hence in order to achieve the expected result by employing this method, it requires modification or the implicit rules must be clarified and made explicit.

\section{Illustrative examples}

\subsection{Non-minimum Phase Plants}

Example 1: Consider the following non-minimum phase plant

$$
P(s)=\frac{k(2-s)}{(s+a)(s+1)}
$$

where the uncertain parameters belong to the set

$$
k \in[1,10] ; \quad a \in[0.1,1]
$$

There is one right half-plane zero. For the sake of simplicity, only the robust stability is considered hereafter and robust performance is not considered. This is justified on the basis that a method must be able to guarantee robust stability if it is to be considered for robust performance. The robust stability requirement can be imposed by

$$
\left|\frac{L(s)}{1+L(s)}\right| \leq 2.1 \text { for all } a \in[0.1,1], k \in[1,10],
$$

where

$$
L(s)=P(s) G(s),
$$

and $G(s)$ is the controller. Condition (3) defines a region around the critical point $(-1,0)$. It is required that the open loop transmission loop under the controller $G(s)$ and uncertainties should not touch this region. In the QFT framework, this condition together with the stability requirement for the nominal plant guarantees the robust 
stability of the closed-loop systems under the described uncertainties with the gain and phase margins defined by (3) (Jayasuriya and Zhao, 1994b; Jayasuriya and Zhao, 1994a). The nominal plant is chosen for the uncertain parameters $a=0.1$ and $k=1$, i.e.,

$$
P_{o}(s)=\frac{2-s}{(s+0.1)(s+1)} .
$$

The plant templates in Nichols chart are shown in Fig. 1 at frequency points $\omega=0.1$, $0.3,1,2,3,6,10,50$ where " $\star$ " denotes the nominal case.

Figure 1 should be here

Figure 2 should be here

Figure 3 should be here

Figure 4 should be here

The robust margin bounds at these frequencies are depicted in Fig. 2. Since the nominal plant (5) can be rewritten as

$$
P_{o}(s)=\frac{s+2}{(s+0.1)(s+1)} \frac{2-s}{2+s}=P_{o}^{\prime}(s) A(s)
$$

where

$$
P_{o}^{\prime}(s)=\frac{s+2}{(s+0.1)(s+1)}
$$

is defined as the new nominal plant. Obviously it is a stable minimum phase plant. The component

$$
A(s)=\frac{2-s}{2+s}
$$

is all-passing with the following properties

$$
|A(j \omega)|=1, \quad \arg (A(j \omega))=-2 \tan ^{-1} 0.5 \omega, \omega \in[0, \infty) .
$$

At frequency $\omega_{i}$, Horowitz and Sidi (1978) pointed out that the new robust bounds for the new nominal plant, $P_{o}^{\prime}$, is obtained by shifting the previous robust bounds by

$$
\phi\left(\omega_{i}\right)=2 \tan ^{-1}\left(0.5 \omega_{i}\right)
$$

The new bounds for the nominal plant $P_{o}^{\prime}(s)$ are depicted in Fig. 3. Horowitz and Sidi (1978) claimed that the QFT controller design for the nominal plant $P_{o}(s)$ is equivalent 
to that for the nominal plant $P_{o}^{\prime}(s)$ with the shifted bounds. So the subsequent design procedure is the same as the QFT design procedure for the minimum phase case developed in Horowitz and Sidi (1972). After manual loop-shaping in the CAD environment of QFT toolbox (Borghesani et al., 1995) in Matlab, a controller

$$
G(s)=\frac{5.845}{s / 1.324+1}
$$

is yielded. The loop transmission $L_{o}^{\prime}(s)=P_{o}^{\prime}(s) G(s)$ is plotted in Fig. 3. All the robust margin bounds are satisfied under this controller. Moreover using the Nichols chart stability criterion (Cohen et al., 1992), the new nominal plant $P_{o}^{\prime}(s)$ under the controller (10) is stable since the loop transmission does not intersect the stability line $R=\left\{(r, \phi): r>0 \mathrm{~dB}, \phi=-180^{\circ}\right\}$. It can also be shown that there is no right half-plane zero-pole cancellation in $G(s) P(s)$ for all $k$ and $a$ within the prescribed ranges (2). The plant family under this controller should be robustly stable. Unfortunately the systems are not robustly stable since, as shown in Fig. 4, the Nyquist plots of the closed-loop systems encircle the point $-1+j 0$. the key issue here is that the stability of the "new" nominal plant does not imply that of the old nominal plant $P(s)$.

\section{$2.2 \quad$ Unstable plants}

The idea for non-minimum phase plants in Horowitz and Sidi (1978) was further extended to the unstable case in Horowitz (1992, Chapter 8). The underlying idea is the same as above, that is, to convert the problem of loop-shaping of an unstable nominal plant to that of a stable minimum phase nominal plant by shifting robust bounds.

Example 2: Consider a simple second order unstable plant

$$
P(s)=\frac{k(s+a)}{s(s-2.5)}
$$

where the uncertain parameters $k$ and $a$ are within the same ranges in (2). This plant has one fixed unstable pole. For simplicity, as in Section 2.1, only robust stability is concerned.

The plant with the parameters $k=1$ and $a=1$ is chosen as the nominal case, giving by

$$
P_{o}(s)=\frac{(s+1)}{s(s-2.5)}
$$


The plant templates at frequencies $\omega_{i}=0.1,0.3,1,2,3,6,10,50$ are plotted in Fig. 5 where " " denotes the nominal plant $P_{o}(j \omega)$. Since this is a plant with negative gain and one integral term, the Nichols plot begins from $-270^{\circ}$ at low frequency. The robust bounds for the nominal plant $P_{o}(s)$ are shown in Fig. 6. Choosing

$$
P_{o}^{\prime}(s)=\frac{(s+1)}{s(s+2.5)}
$$

as the new nominal plant, the relationship between the nominal plant $P_{o}(s)$ and the new nominal plant $P_{o}^{\prime}(s)$ is given by

$$
\left|P_{o}(j \omega)\right|=\left|P_{o}^{\prime}(j \omega)\right|
$$

and

$$
\arg P_{o}(j \omega)=\arg P_{0}^{\prime}(j \omega)-180^{\circ}+2 \tan ^{-1}(0.4 \omega), \omega \in[0, \infty) .
$$

Figure 5 should be here

Figure 6 should be here

Figure 7 should be here

Figure 8 should be here

Thus the bounds for the new nominal plant $P_{o}^{\prime}(s)$, as shown in Fig. 7, are yielded by shifting the bounds of the nominal plant $P_{o}(s)$ right by

$$
180^{\circ}-2 \tan ^{-1}(0.4 \omega), \text { for all } \omega \in[0, \infty)
$$

The problem now becomes to design a controller such that the loop transmission $L^{\prime}(s)$ satisfies all the bounds in Fig. 7 for the plant $P_{o}^{\prime}(s)$. The Nichols plot of the loop transmission $L^{\prime}(s)$ after the loop shaping is depicted in Fig. 7 and the corresponding controller is given by

$$
G(s)=\frac{1.571}{s / 0.2616+1} .
$$

Once again all the robust bounds are satisfied and the new nominal closed-loop system is stable under this controller. The controller $G(s)$ should stabilise the uncertain plant (11). However as shown in Fig. 8, the closed loop system is not robustly stable. The Nyquist plots encircle the point $-1+j 0$ in the clockwise direction once and since the 
open loop plant has one unstable pole, the closed-loop system has two unstable poles. It should be noted that the Nyquist plot of the plant $P_{o}^{\prime}(s)$ is also drawn in Fig. 8 and it does not circle the point $-1+j 0$ since the low frequency gain of $P_{o}^{\prime}(s)$ is positive and there is no unstable pole in $P_{o}^{\prime}(s)$. So the controller $G(s)$ in (15) stabilises the new nominal plant $P_{o}^{\prime}(s)$ but does not stabilise the uncertain plant $P(s)(11)$. The relationship between the stability of the new nominal plant $P_{o}^{\prime}(s)$ and the previours nominal plant should be examined.

\section{Robust bounds and stability for the new nominal plant}

In Section 2, two examples are presented to show that the design method for nonminimum phase and unstable plants developed in Horowitz and Sidi (1978) and Horowitz (1992) may not work. It is believed by some researchers in QFT community that in loop shaping, the open loop transmission $L(j \omega)$ must pass the corresponding QFT bounds on the right hand. This rule, however, has not been clearly stated and, more importantly, there is no any mathematical justification on it. In this section, the relationship between an unstable and/or non-minimum phase nominal plant and a "new" stable, minimum phase nominal plant is examined carefully. Some implicit rules in Horowitz and Sidi $(1978 ; 1992)$ are clarifies and an improved method is given.

\subsection{QFT bounds}

Consider a SISO uncertain plant

$$
P(s)=\frac{N(s) \hat{N}(-s)}{D(s) \hat{D}(-s)}
$$

where $\hat{D}(-s)$ and $\hat{N}(-s)$ denote the parts with right half-plane poles and zeros explicitly.

Specifications in the QFT formulation are generally expressed by the following three item

1. Robust Stability Margin

$$
\left|\frac{L(s)}{1+L(s)}\right| \leq \gamma
$$

2. Tracking Performance

$$
a(\omega) \leq\left|\frac{F(j \omega) L(j \omega)}{1+L(j \omega)}\right| \leq b(\omega)
$$


3. Disturbance Attenuation Performance

$$
\left|\frac{P(j \omega)}{1+L(j \omega)}\right| \leq d(\omega)
$$

where

$$
L(s)=P(s) G(s)
$$

and $G(s)$ and $F(s)$ are the controller and the prefilter respectively. In addition, it is important to note that to ensure robust stability of the closed loop systems, the nominal closed-loop system must be stable. So the QFT design problem is to find a controller $G(s)$ and a prefilter $F(s)$ such that conditions (17), (18) and (19) are satisfied and an arbitrarily chosen nominal plant is stabilised. An arbitrarily chosen nominal plant within the plant family is given by

$$
P_{o}(s)=\frac{N_{o}(s) \hat{N}_{o}(-s)}{D_{o}(s) \hat{D}_{o}(-s)} .
$$

If

$$
A(s)=\frac{\hat{N}_{o}(-s) \hat{D}_{o}(s)}{\hat{N}_{o}(s) \hat{D}_{o}(-s)},
$$

then the new nominal plant is defined as

$$
P_{o}^{\prime}(s)=P_{o}(s) A(s)^{-1}=\frac{N_{o}(s) \hat{N}_{o}(s)}{D_{o}(s) \hat{D}_{o}(s)} .
$$

Obviously $P_{o}^{\prime}(s)$ is a stable minimum phase plant. Furthermore we have

$$
L(s)=P(s) G(s)=P^{\prime}(s) G(s) A(s)=L^{\prime}(s) A(s)
$$

where

$$
P^{\prime}(s)=\frac{N(s) \hat{N}(-s) \hat{N}_{o}(s) \hat{D}_{o}(-s)}{D(s) \hat{D}(-s) \hat{N}_{o}(-s) \hat{D}_{o}(s)} .
$$

and

$$
L^{\prime}(s)=P^{\prime}(s) G(s)
$$

Since $A(s)$ defined in (21) is all-passing, it gives

$$
|A(j \omega)|=1 \text { for all } \omega \in[0, \infty) .
$$

This implies

$$
|1+L(s)|=\left|1+L^{\prime}(s) A(s)\right|=\left|A(s)^{-1}+L^{\prime}(s)\right| .
$$


The stability margin in (17) becomes

$$
\left|\frac{L(j \omega)}{1+L(j \omega)}\right|=\left|\frac{L^{\prime}(j \omega)}{A(j \omega)^{-1}+L^{\prime}(j \omega)}\right| \leq \gamma
$$

$L^{\prime}(j \omega)$ under the allowable uncertainties must satisfy condition (28). That is, on the Nichols chart the stability margin bounds on $L(j \omega)$ defined in (17) are regions with the centre $\left(0^{\circ}, 0 \mathrm{db}\right)$ but the stability margin bounds on $L^{\prime}(j \omega)$ defined in (28) are regions with the centre of $\left(\arg \left(A(j \omega)^{-1}\right), 0 \mathrm{db}\right)$ which varies with the frequency $\omega$. To show it, note that

$$
L(j \omega)=\frac{P(j \omega)}{P_{o}(j \omega)} L_{o}(j \omega)
$$

It follows that the robust stability bound for the nominal case $L_{o}(j \omega)$ is calculated from the inequality

$$
\left|\frac{\frac{P(j \omega)}{P_{o}(j \omega)} L_{o}(j \omega)}{1+\frac{P(j \omega)}{P_{o}(j \omega)} L_{o}(j \omega)}\right| \leq \gamma .
$$

Similarly, for the new nominal loop transmission, $L_{o}^{\prime}(j \omega)$, we have

$$
\left|\frac{\frac{P(j \omega)}{P_{o}(j \omega)} L_{o}^{\prime}(j \omega) A(j \omega)}{1+\frac{P(j \omega)}{P_{o}(j \omega)} L_{o}^{\prime}(j \omega) A(j \omega)}\right| \leq \gamma .
$$

At a prescribed frequency $\omega_{i}$, the bound for $L_{o}\left(j \omega_{i}\right)$ is derived from condition $(29)$. Let

$$
L_{o}\left(j \omega_{i}\right)=r e^{j \phi}
$$

It follows from (29) that

$$
\begin{gathered}
\left|\frac{\frac{P\left(j \omega_{i}\right)}{P_{o}\left(j \omega_{i}\right)} r e^{j \phi}}{1+\frac{P\left(j \omega_{i}\right)}{P_{o}\left(j \omega_{i}\right)} r e^{j \phi}}\right| \\
=\left|\frac{\frac{P\left(j \omega_{i}\right)}{P_{o}\left(j \omega_{i}\right)} r e^{j\left(\phi-\arg A\left(j \omega_{i}\right)\right)} A\left(j \omega_{i}\right)}{1+\frac{P\left(j \omega_{i}\right)}{P_{o}\left(j \omega_{i}\right)} r e^{j\left(\phi-\arg A\left(j \omega_{i}\right)\right)} A\left(j \omega_{i}\right)}\right| .
\end{gathered}
$$

Now let

$$
L_{o}^{\prime}(j \omega)=r^{\prime} e^{j \phi}
$$

Substituting it into (30) and then comparing with (31) yields

$$
\phi^{\prime}=\phi-\arg A(j \omega), r^{\prime}=r
$$

This implies that if the bound on the magnitude $\left|L_{o}\left(j \omega_{i}\right)\right|$ is $r_{o}$ at the phase $\phi$, then the bound on the magnitude of $\left|L_{o}^{\prime}\left(j \omega_{i}\right)\right|$ is also $r_{o}$ at the phase $\phi-\arg A\left(j \omega_{i}\right)$. Hence the bound on $L_{o}^{\prime}\left(j \omega_{i}\right)$ can be obtained by shifting the bound on $L_{o}(j \omega)$ by -arg $A\left(j \omega_{i}\right)$. 
Similar conditions can be found for the bounds of tracking performance and disturbance rejection.

Following

$$
\left|\frac{F(j \omega) L(j \omega)}{1+L(j \omega)}\right|=\left|\frac{F(j \omega) L^{\prime}(j \omega)}{A(j \omega)^{-1}+L^{\prime}(j \omega)}\right|,
$$

condition (18) becomes

$$
a(\omega) \leq\left|\frac{F(j \omega) L^{\prime}(j \omega)}{A(j \omega)^{-1}+L^{\prime}(j \omega)}\right| \leq b(\omega)
$$

Similarly, the disturbance attenuation performance (19) can be written as

$$
\left|\frac{P^{\prime}(j \omega)}{A(j \omega)^{-1}+L^{\prime}(j \omega)}\right| \leq d(\omega) .
$$

It can also be shown that the bounds imposing on $L^{\prime}(j \omega)$ by (33) and (34) are the same as the bounds imposing on $L(j \omega)$ by (18) and (19) but with horizontal shift of $-\arg A(j \omega)$, which is similar to the stability margin bounds (17).

The bounds for stability margin, tracking and disturbance attenuation for the stable minimum phase nominal plant $P_{o}^{\prime}\left(j \omega_{i}\right)$ are therefore the same as that for the unstable and/or non-minimum phase nominal plant $P_{o}\left(j \omega_{i}\right)$ but with a horizontal (in the Nichols plane) shift of phase $-\arg \left(A\left(j \omega_{i}\right)\right)$ at frequency $\omega_{i}$. That is, the bounds for the new nominal plant $P_{o}^{\prime}\left(j \omega_{i}\right)$ are obtained by shifting the bounds for the nominal plant $P_{o}\left(j \omega_{i}\right)$ by $-\arg \left(A\left(j \omega_{i}\right)\right)$. This conclusion is the same as that of Horowitz and Sidi (1978) and Horowitz (1992) but a strictly mathematical derivation is not given there. However, an important requirement, the stability of the nominal closed-loop system, is not clearly specified by Horowitz and Sidi (1978) and Horowitz (1992).

\subsection{Stability analysis}

Next a stability criterion for the new nominal plant $P_{o}^{\prime}(s)$ is proposed. First some necessary preliminaries are given. The stability line in Nyquist plot is defined as

$$
R_{c}=:\{(x, y): y=0, x<-1\} \text {. }
$$

which is shown in Figure 9. According to the relationship between the Nyquist plot and Nichols plot, as shown in Figure 9, the stability line in single sheet Nichols chart is given by

$$
R_{n}=:\left\{(\phi, r): \phi=-180^{\circ}, r>0\right\}
$$


and the stability lines in the multiple sheet Nichols chart are given by

$$
R_{n}=\left\{(\phi, r): \phi=-(2 q+1) 180^{\circ}, r>0, q=0, \pm 1, \pm 2, \ldots\right\} .
$$

A crossing occurs when the plot of $L(s)$ intersects the stability line $R_{c}$. The crossing is said to be positive if the direction is upward, and negative otherwise, as shown in Fig 9.

Lemma(Graphical stability criterion on Nyquist plot (Vidyasagar et al., 1988)): For a plant with $n$ right half plane poles, the closed loop system is stable if and only if the number of positive crossings minus the number of negative crossings is $n$ and $L(j \omega)$ does not intersect with the point $-1+j 0$.

The Nyquist plot does not intersect the point $(-1+j 0)$ implies

$$
1+L(j \omega) \neq 0
$$

It follows from (23) that

$$
L^{\prime}(j \omega) \neq-A(j \omega)
$$

Thus the critical point moves with frequency instead of the fixed point $-1+j 0$. Noting that $\left|A^{-1}(j \omega)\right|=1$, the critical point moves on the boundary of the unit circle as shown in Fig. 10.

Figure 9 should be here

Moreover, according to the positive crossing and negative crossing definitions in Fig. 9, a crossing occurs at frequency $\omega_{i}$ if and only if

$$
1+L\left(j \omega_{i}\right)=-k
$$

where $k$ is an arbitrary positive real. This implies

$$
L^{\prime}\left(j \omega_{i}\right)=-(k+1) A^{-1}\left(j \omega_{i}\right)
$$

Therefore $L(j \omega)$ intersects the stability line $R_{c}$ in (35) at frequency $\omega_{i}$ is equivalent to that $L^{\prime}(j \omega)$ intersects the line $-(k+1) A^{-1}(j \omega)$ for $k>0$ at frequency $\omega_{i}$. The stability lines for $L^{\prime}(j \omega)$ with respect to the stability line $R_{c}$ in $(35)$ for $L(j \omega)$ are shown in Fig. 10. At frequency $\omega_{i}$, the stability line for $L^{\prime}(s)$ in the Nyquist plot is given by

$$
\begin{gathered}
R_{c}(\omega)=:\{(x, y): x=-(k+1) \cos (\arg (-A(j \omega)), \\
y=-(k+1) \sin (\arg (-A(j \omega)), \quad k>0\} .
\end{gathered}
$$


This implies the stability line for $L^{\prime}(s)$ moves with frequency instead of a fixed negative real axis for $L(s)$. In the Nichols chart the stability lines become a series of vertical lines as shown in Fig. 10, given by

$$
\begin{aligned}
R_{n}(\omega)= & \left\{(\phi, r): \phi=-(2 q+1) 180^{\circ}+\arg \left(A^{-1}(j \omega)\right),\right. \\
& r>0, q=0, \pm 1, \pm 2, \ldots\}
\end{aligned}
$$

Following the analysis in the above, a crossing occurs for $L^{\prime}(j \omega)$ only when the plot of $L^{\prime}(j \omega)$ intersects the stability line $R_{n}(\omega)$ at the same frequency $\omega$. The above result is summarised in Theorem.

Figure 10 should be here

Definition: Suppose that at any $\omega, L(\omega)$ and $R(\omega)$ are a point and a line in the complex plane respectively. We say, with $\omega$ from 0 to $\infty$, the curve $L(\omega)$ crosses the line $R\left(\omega_{i}\right)$ if $L\left(\omega_{i}\right)$ is on the line $R\left(\omega_{i}\right)$ at $\omega_{i}$. Moreover we say the curve $L(\omega)$ crosses the line $R\left(\omega_{i}\right)$ with positive direction if the the curve $L(\omega)$ intersects the line $R\left(\omega_{i}\right)$ with the clockwise direction, and negative otherwise.

Theorem: For a plant $L(s)$ with $n$ right half plane poles, the closed-loop system is stable if and only if $L^{\prime}(j \omega)$ defined in (23) does not intersect the point $-A(j \omega)$ at the same frequency $\omega$ and the net number of positive and negative crossings on the stability line $R_{n}(\omega)$ in (41) at the same $\omega$ is $n$.

\subsection{Design Guidelines in QFT}

When the loop shaping problem in QFT for a non-minimum phase and/or unstable nominal plant, $P_{o}(s)$, is transformed to that for a stable minimum phase nominal plant, $P_{o}^{\prime}(s)$, beside the bounds for the nominal plant $P_{o}(s)$ should be moved with $-A\left(j \omega_{i}\right)$ at the frequency $\omega_{i}$, stability of the new nominal plant $P_{o}^{\prime}(s)$ must be reconsidered. That is, not only the robust bounds but also the stability line must be shifted with the frequency. The new stability criterion is presented in Theorem. It is important to notice that in the loop shaping procedure, whether or not the loop transmission $L^{\prime}(j \omega)$ must intersect the stability line $R(\omega)$ depends on the number of unstable poles in the original nominal plant, $P_{o}(s)$, rather than that in the new nominal plant, $P_{o}^{\prime}(s)$. For example, if $P_{o}(s)$ 
has one unstable pole, to ensure stability of the nominal closed-loop system, the loop transmission $L^{\prime}(j \omega)$ must intersect the stability line $R(\omega)$ with one positive crossing although it has no unstable poles.

It has become customary that in QFT and other control design methods to use only half of the Nyquist plot. Because of conjugate symmetry, each crossing of the half plot is equal to two crossing of the complete plot. If the half plot crosses the stability line at the beginning, it's counted as one half crossing.

Since the stability line is connected to the QFT bounds at each frequency $\omega_{i}$, it is also shifted with the same horizontal distance as the QFT bounds. For a non-minimum phase plant, it's easy to find that a necessary and sufficient condition for stability is that the net number of positive and negative crossings is zero and the Nyquist plot of the open loop transmission passes the corresponding QFT bounds on the right hand. For an unstable system, as shown in Theorem, stability depends on the number of unstable poles and the net number of the positive and negative crossings.

To summary, some design guidelines of Horowitz and Sidi method for non-minimum phase and unstable plants are clarified as follows:

1. When transferring the loop shaping problem for a non-minimum phase and/or unstable nominal plant $P_{o}(s)$ to that for a stable, minimum phase nominal plant $P_{o}^{\prime}(s)$, the stability line must be shifted with the same distance as robust stability and performance bounds.

2. In the loop shaping, to ensure stability of the closed-loop system, the net number of the positive and negative crossings of the open loop transmission $L^{\prime}(j \omega)$ depends on the number of unstable poles in the original nominal plant $P_{o}(s)$, rather than that in the new nominal plant $P_{o}^{\prime}(s)$

3. For a non-minimum phase plant, a necessary and sufficient condition for the nominal system to be stable is that the open loop transmission $L^{\prime}(j \omega)$ does not intersect the point $-A(j \omega)$ at the same frequency and the net number of positive and negative crossings is zero. Moreover in this case, the Nyquist plot of the open loop transmission passes the corresponding QFT bounds on the right hand.

4. For an uncertain plant with $n$ unstable poles, a necessary and sufficient condition for the closed-loop system is stable is that the half of the Nyquist plot of the 
open loop transmission $L^{\prime}(j \omega)$ does not intersect the point $-A(j \omega)$ at the same frequency and the net number of positive and negative crossings on the stability line is $n / 2$.

\section{Examples revisited}

\subsection{Non-minimum Phase Plants}

Example 1 in (1) is a non-minimum phase plant without unstable poles. When the loop shaping problem for the non-minimum phase nominal plant, $P_{o}$ in $(5)$, is transferred to that for the minimum phase nominal plant, $P_{o}^{\prime}$ in $(7)$, the stability line should also be shifted with the same distance as the robust bounds in Figure 3. It is easy to find that the open loop transmission crosses the stability line one time in Figure 3. Hence from the stability result in this paper, the nominal system is unstable. This can also be shown by that the loop transmission passes the corresponding robust bound on the left hand.

\subsection{Unstable Plants}

Figure 11 should be here

Figure 12 should be here

Similar to Example 1, Example 2 in Section 2 is revisited here. Shifting the robust margin bounds and the stability line simultaneously yields the robust margin bounds and the stability lines in Fig. 11. Now we check the loop shaping result in Figure 7 under the controller (15). When $\omega$ is zero, the stability line is shifted to 0 deg phase. There is one negative half crossing at the beginning of the Nyquist plot of the open loop transmission. There is no other crossing. Hence the number of crossings of the complete Nyquist is -1 . This plant has one unstable pole. Hence the nominal system is unstable.

Now we redesign the controller for Example 2 by taking into account the new stability

requirement. For this special plant, only a simple gain controller is needed to satisfy the robust margin bounds and the stability requirement of the nominal case, given by

$$
G(s)=6.582
$$


As shown in Figure 11, at the beginning of the Nyquist plot, the loop transmission $L^{\prime}(j \omega)$ has one negative half crossing. Then it intersects the stability line with one positive crossing. (Chen and Ballance (1998) has developed a series of simple rules to calculate the net number of crossing for complicated plants). The net number of the positive and negative crossings is $2 \times(1-1 / 2)=1$. The nominal plant under this controller is stable since the plant $P_{o}(s)$ has one unstable pole. So it implies the closed-loop systems are robustly stable and with the stability margin defined in (3). The Nyquist plots of the closed-loop system are also given in Fig. 12, which also show that it is robustly stable.

\section{Conclusion}

This paper revisits the QFT design method for non-minimum phase and unstable plants developed by Horowitz and Sidi (1978) and Horowitz (1992). Motivated by two examples including unstable and non-minimum phase plants, we show that the closed-loop systems under the controller resulting from the existing Horowitz and Sidi method may not be robustly stable and the guidance in the existing method should be clarified or modified. By the rigorous mathematical induction, it is shown that the reason is that the stability requirement of the new nominal plant is not clearly specified. It is pointed out that the stability line of the new nominal plant varies with frequency instead of a fixed negative real axis. The mathematically sound basis for transferring the robust bounds of an unstable and/or non-minimum phase nominal plant into that of a stable minimum phase nominal plant is also established. This paper takes a further step toward establishing the mathematical justification of the current QFT design procedure and providing clear guidelines for control system designers wishing to use QFT.

\section{Acknowledgement}

This work was financially supported by UK Engineering and Physical Science Research Council under the grant No. GR/J92989. 


\section{References}

Ballance, D. J. (1992). Comments on the papers "A new approach to optimum loop synthesis" and "On the determination of plant variation bounds for optimum loop synthesis". Int. J. Control 55(1), 241-248.

Ballance, D. J. and P. J. Gawthrop (1992). QFT, the UHB, and the choice of the template nominal point. In: Proceedings of the Symposium on Quantitative Feedback Theory (C. N. Houpis and P.R. Chandler, Eds.). Dayton, Ohio, U.S.A.

Bode, H. W. (1945). Network Analysis and Feedback Amplifier Design. van Nostrand. New York.

Borghesani, C., Y. Chait and O. Yaniv (1995). Quantitative Feedback Theory Toolbox User Manual. The Math Work Inc.

Chait, Y., M. S. Park and M. Steinbuch (1994). Design and implementation of a QFT controller for a compact disc player. In: Proceedings of the 1994 American Control conference. Vol. 3. Baltimore, Maryland, U.S.A.. pp. 3204-3208.

Chen, Wen-Hua and Donald J. Ballance (1998). Stability analysis on the Nichols chart and its application in QFT. Technical Report CSC-98013. Centre for Systems \& Control, University of Glasgow.

Chen, Wen-Hua and Donald J. Ballance (1999). Plant template generation of uncertain plants in Quantitative Feedback Theory. ASME Journal of Dynamic Systems, Measurement and Control 121(3), 358-364.

Cohen, N., Y. Chait, O. Yaniv and C. Borghesani (1992). Stability analysis using nichols charts. In: Proceeding of teh Symposium on Quantitative Feedback Theory (C.N. Houpis and P.R. Chandler, Eds.). Dayton, Ohio, U.S.A.. pp. 80-103.

Doyle, J. C. (1986). Quantitative feedback theory (QFT) and robust control. In: Proceedings of the 1986 American Control Conference. Seattle, Washington, U.S.A.

Horowitz, I. M. (1963). Synthesis of Feedback Systems. Academic Press.

Horowitz, I. M. (1992). Quantitative Feedback Design Theory (QFT). Vol. 1. QFT Publications, 4470 Grinnel Ave., Boulder, Colorado 80303, USA. 
Horowitz, I. M. and M. Sidi (1972). Synthesis of feedback systems with large plant ignorance for prescribed time-domain tolerances. Int. J. Control 16, 287-309.

Horowitz, I. M. and M. Sidi (1978). Optimum synthesis of non-minimum phase feedback system with plant uncertainty. Int. J. Control 27, 361-386.

Houpis, C. H. (1995). Quantitative Feedback Theory (QFT) For The Engineer: A Paradigm for the Design of Control Systems for Uncertain Nonlinear Plants. Wright Laboratory.

Jayasuriya, S. and Y. Zhao (1994a). Stability of Quantitative Feedback Design and the exitence of robust QFT controllers. International Journal of Robust and Nonlinear Control 4(1), 21-46.

Jayasuriya, S. and Yongdong Zhao (1994b). Robust stability of plants with mixed uncertainties and quantitative feedback theory. AMSE Journal Dynamic Systems, Measurement, and Control 116(2), 10-16.

Nordgren, R. E., O. D. I. Nwokah and M. A. Franchek (1994). New formulations for quantitative feedback theory. International Journal of Robust and Nonlinear Control 4, 47-64.

Pachter, M. and C. H. Houpis (1997). A full envelope flight control system design, including aerodynamic control effector failures accommodation, using QFT. In: Proceedings of the Symposium on Quantitative Feedback theory and other Frequency Domain Methods and Applications (L. Petropoulakis and W.E Leithead, Eds.). Glasgow, Scotland. pp. 45-54.

Vidyasagar, M., R. K. Bertschmann and C. S. Sallaberger (1988). Some simplifications of the graphical nyquist criterion. IEEE Trans. on Automatic Control 33(3), 301-205.

Yaniv, O. and I. M. Horowitz (1987). Quantitative feedback theory-reply to criticisms. Int. J. Control 46(3), 945-962. 


\section{List of Figures}

1 Plant templates and the nominal plant for Example 1 . . . . . . . . . 19

2 Robust margin bounds of Example 1: the nominal plant $P_{o}(s) \ldots \ldots$

3 Robust margin bounds for the new nominal plant $P_{o}^{\prime}(s)$ and loop-shaping result for Example 1: the QFT bounds from the left to the right and the circles on the loop transmission from the top to the bottom corresponding to frequencies $\omega_{i}=0.1,0.3,1,2,3,6,10,50 \ldots \ldots 21$

4 Nyquist plots under the resulting controller for Example 1 . . . . . . 22

$5 \quad$ Plant templates and the nominal plant for Example $2 \ldots \ldots 23$

6 Robust margin bounds for Example 2: nominal plant $P_{o}(s) \ldots \ldots 24$

$7 \quad$ Robust margin bounds for the new nominal plant $P_{o}^{\prime}(s)$ and loop-shaping for Example 2: the QFT bounds from the right to the left and the circle points on the loop transmission from the top to the bottom corresponding to frequencies $\omega_{i}=0.1,0.3,1,2,3,6,10,50 \ldots \ldots 25$

8 Nyquist plots under the resulting controller for Example 2 . . . . . . . 26

9 The Definition of Positive and Negative Crossings . . . . . . . . . . . . 27

10 Stability lines on Nyquist plot and Nichols chart for the new nominal plant 28

11 Loop-shaping for Example $2 \ldots \ldots \ldots$. . . . . . . . . . . 29

12 Nyquist plots under the new controller for Example $2 \ldots$. . . . . . . 30 


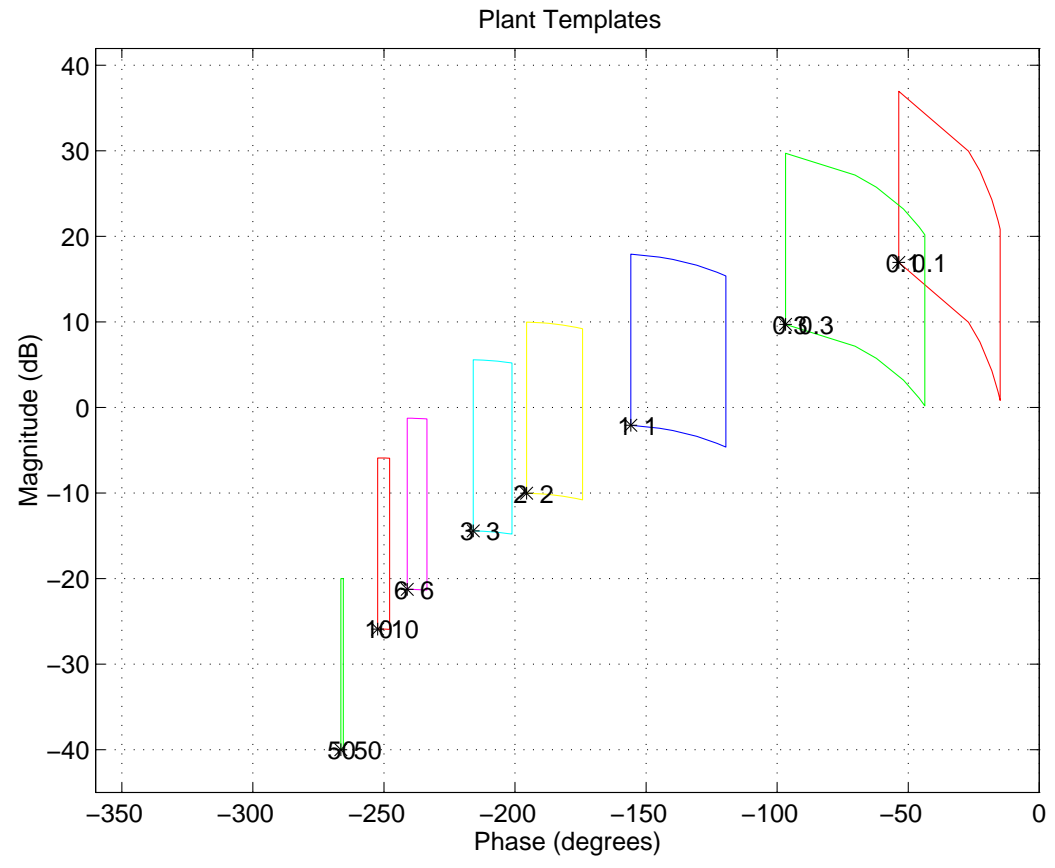

Figure 1: Plant templates and the nominal plant for Example 1 


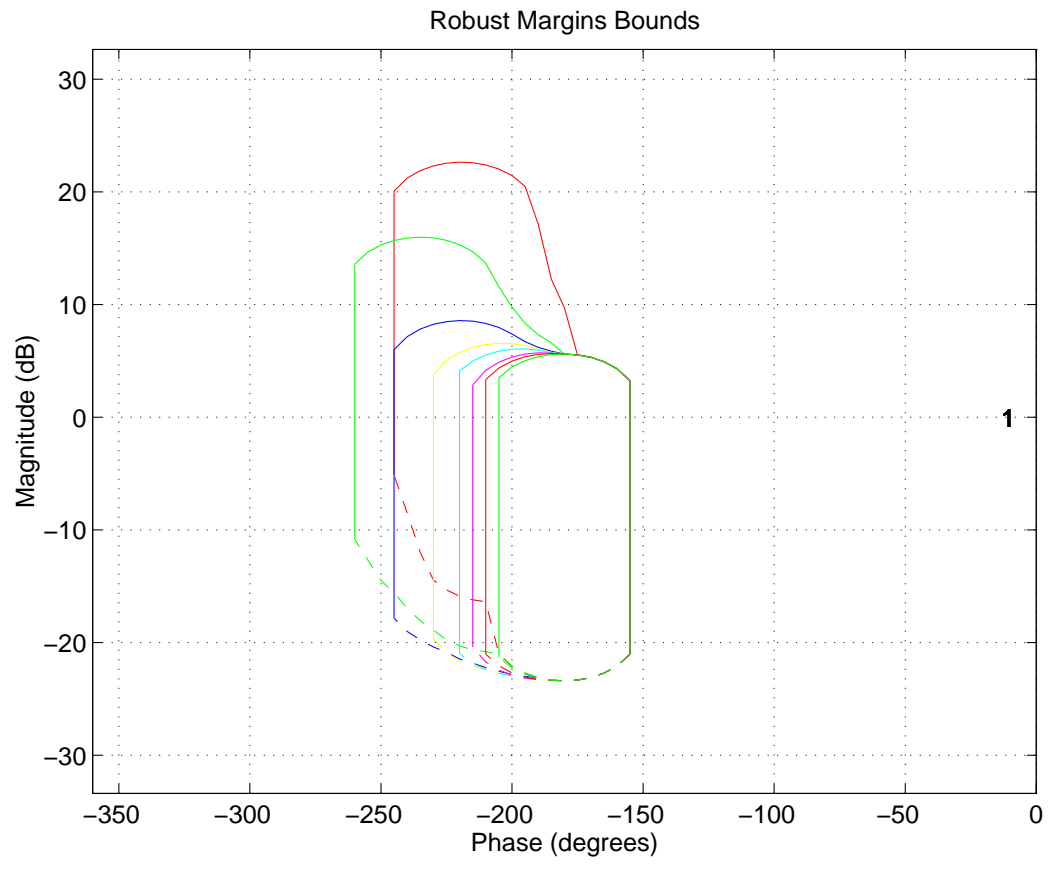

Figure 2: Robust margin bounds of Example 1: the nominal plant $P_{o}(s)$ 


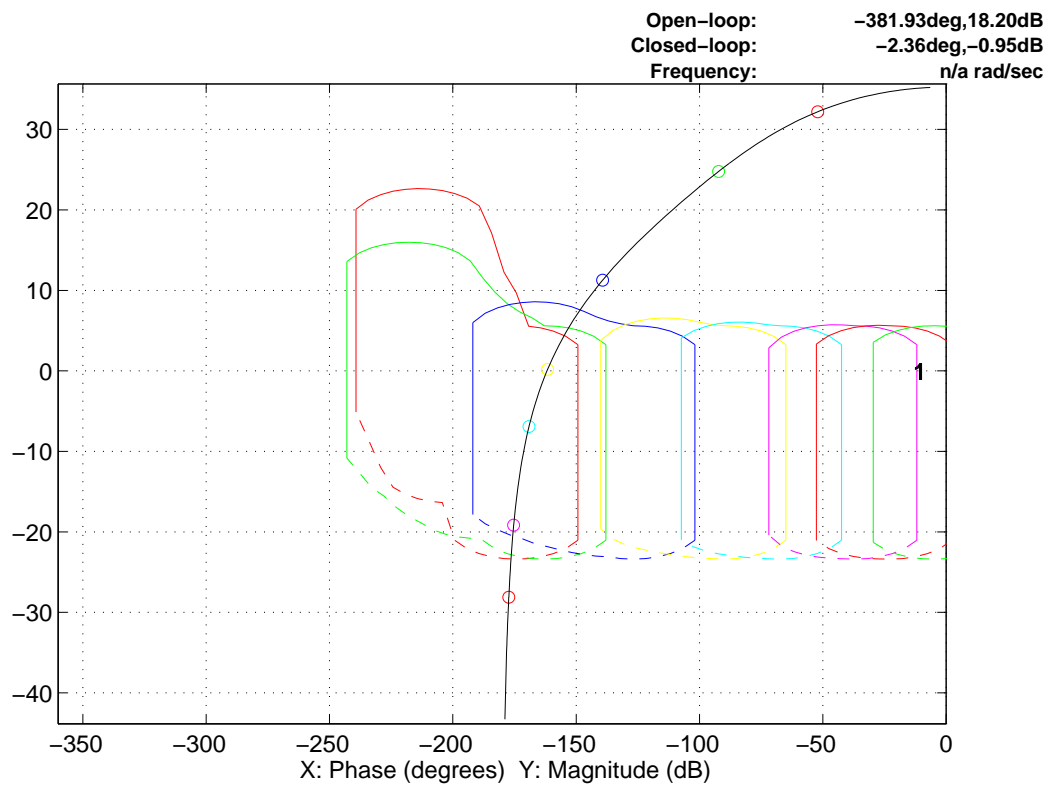

Figure 3: Robust margin bounds for the new nominal plant $P_{o}^{\prime}(s)$ and loop-shaping result for Example 1: the QFT bounds from the left to the right and the circles on the loop transmission from the top to the bottom corresponding to frequencies $\omega_{i}=0.1$, $0.3,1,2,3,6,10,50$ 


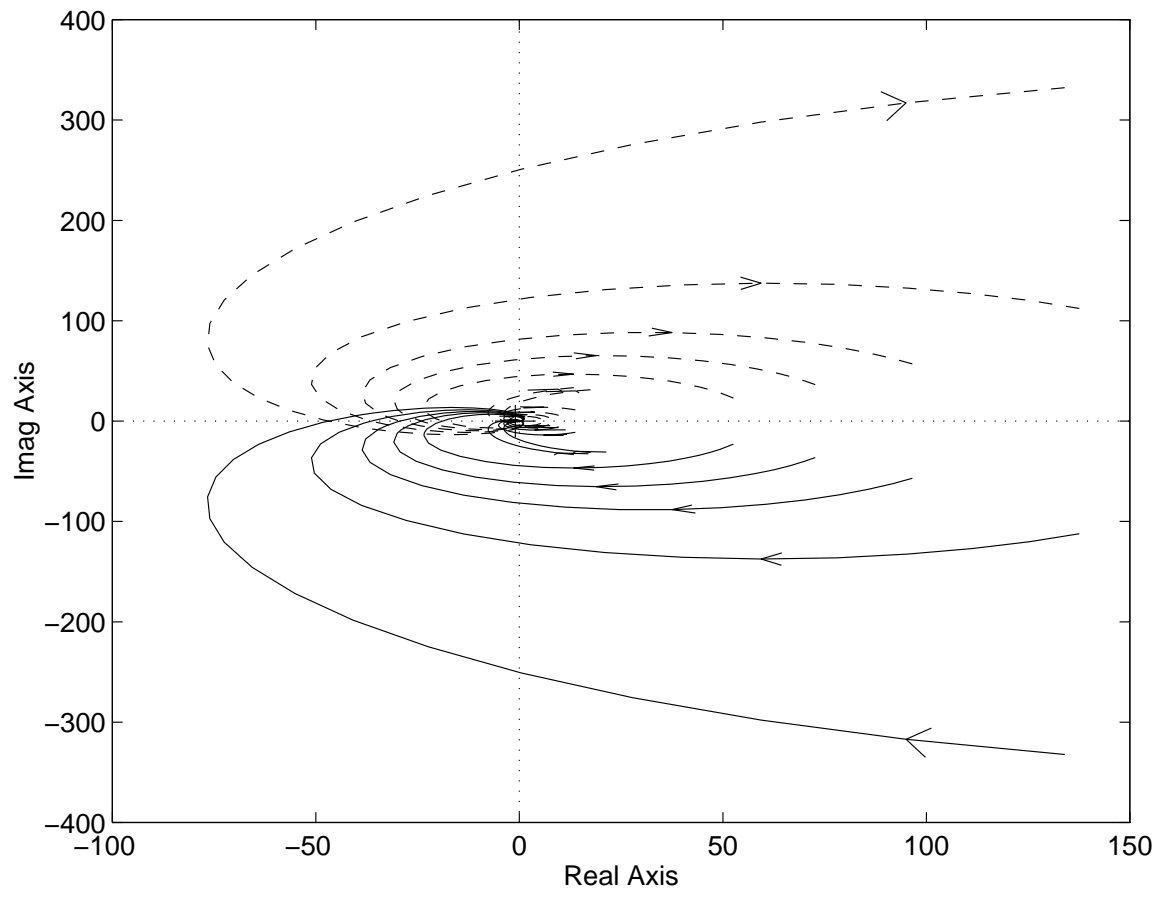

Figure 4: Nyquist plots under the resulting controller for Example 1 


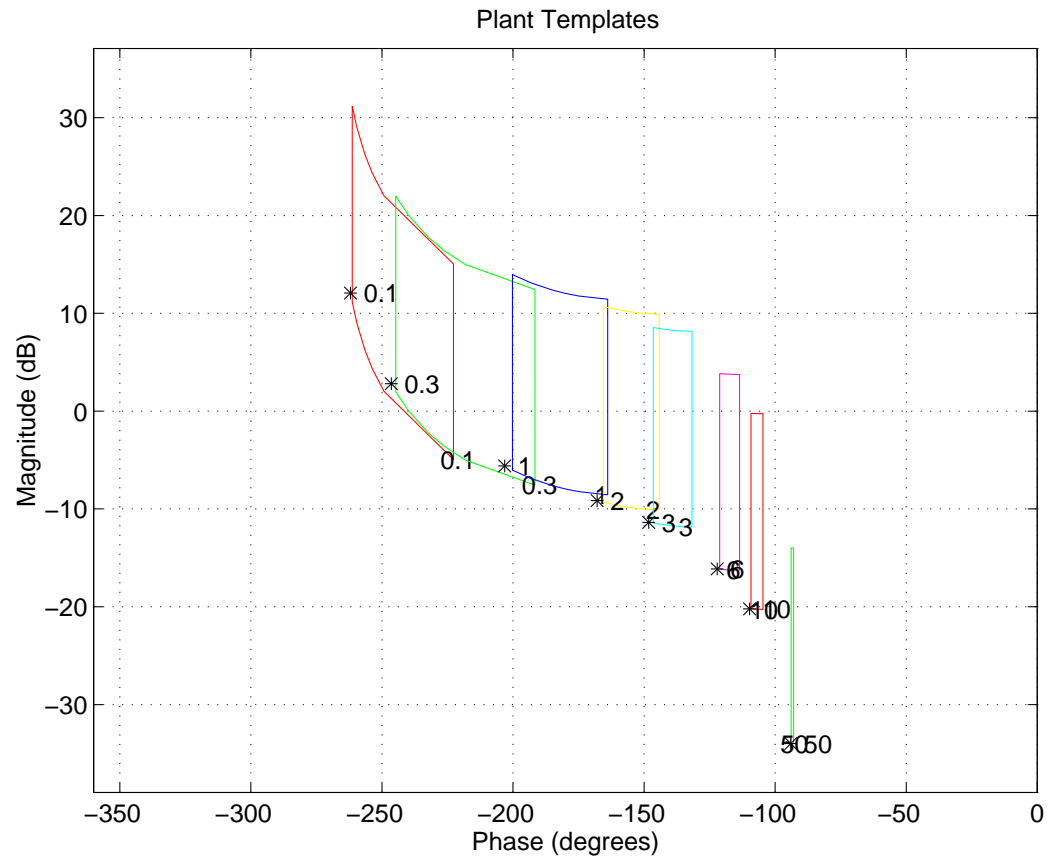

Figure 5: Plant templates and the nominal plant for Example 2 


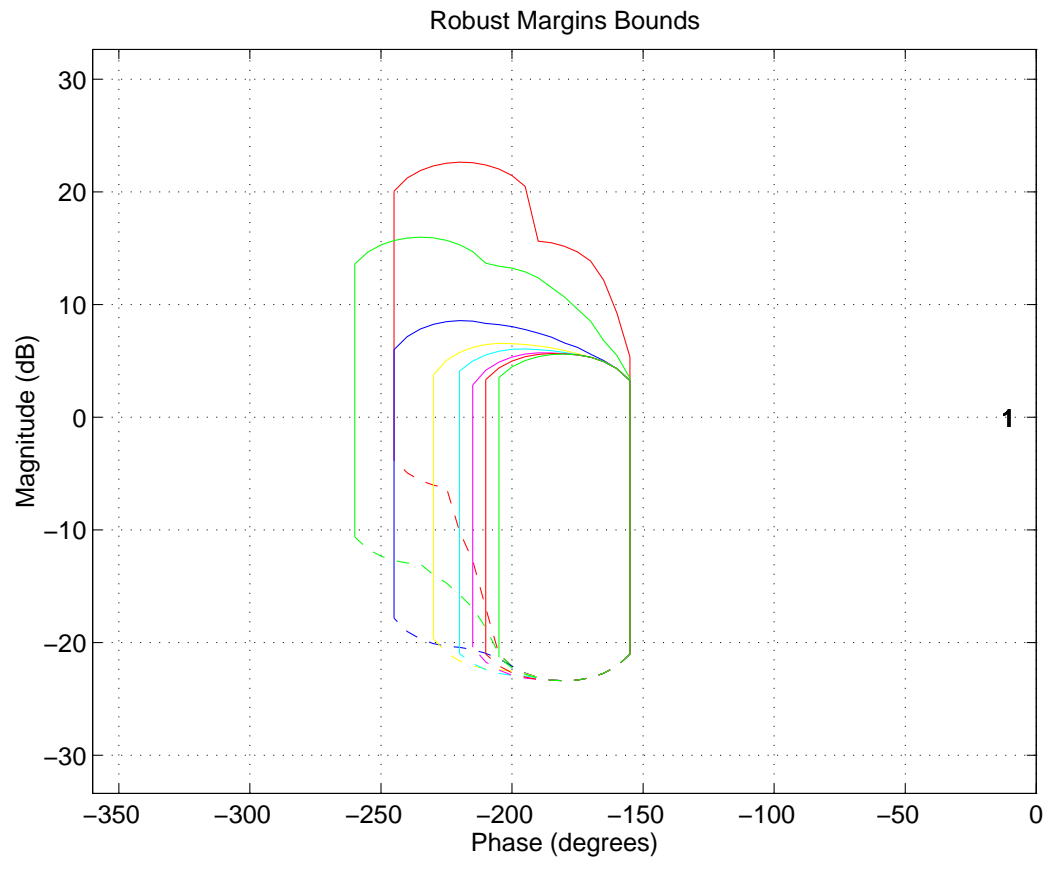

Figure 6: Robust margin bounds for Example 2: nominal plant $P_{o}(s)$ 


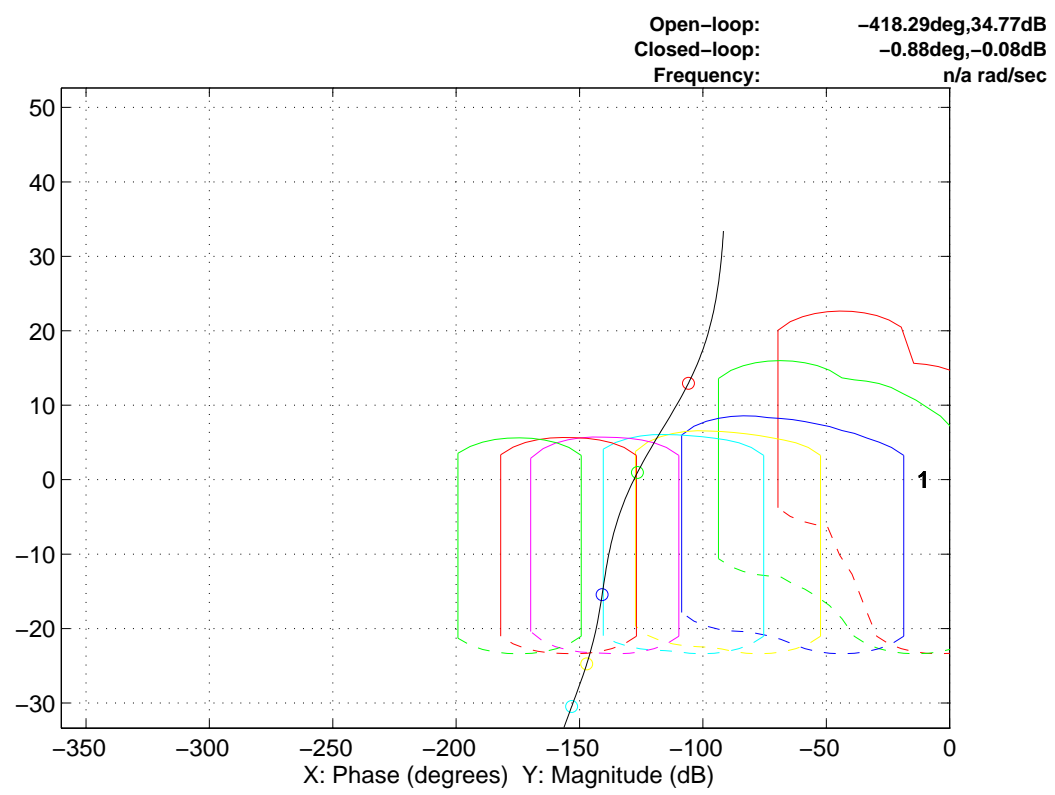

Figure 7: Robust margin bounds for the new nominal plant $P_{o}^{\prime}(s)$ and loop-shaping for Example 2: the QFT bounds from the right to the left and the circle points on the loop transmission from the top to the bottom corresponding to frequencies $\omega_{i}=0.1,0.3,1$, $2,3,6,10,50$ 


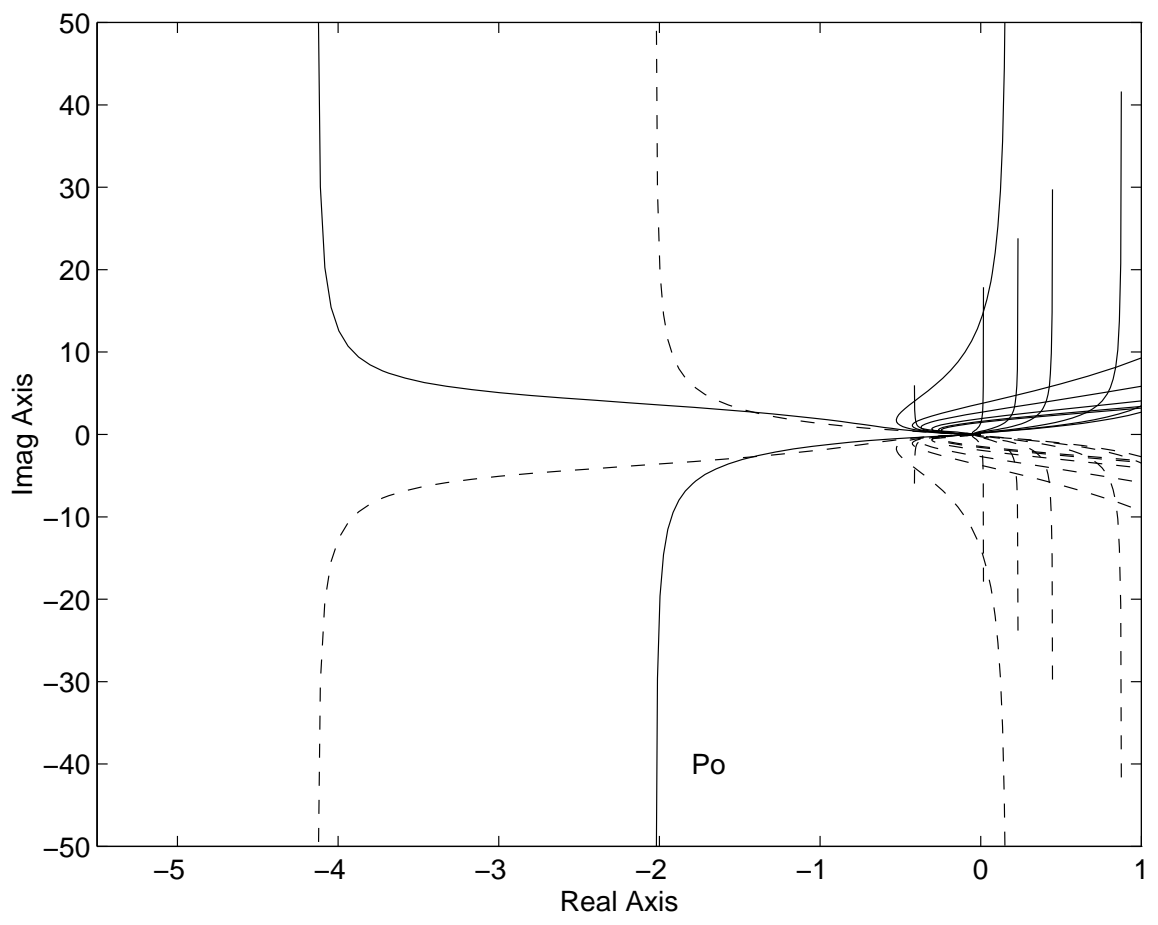

Figure 8: Nyquist plots under the resulting controller for Example 2 


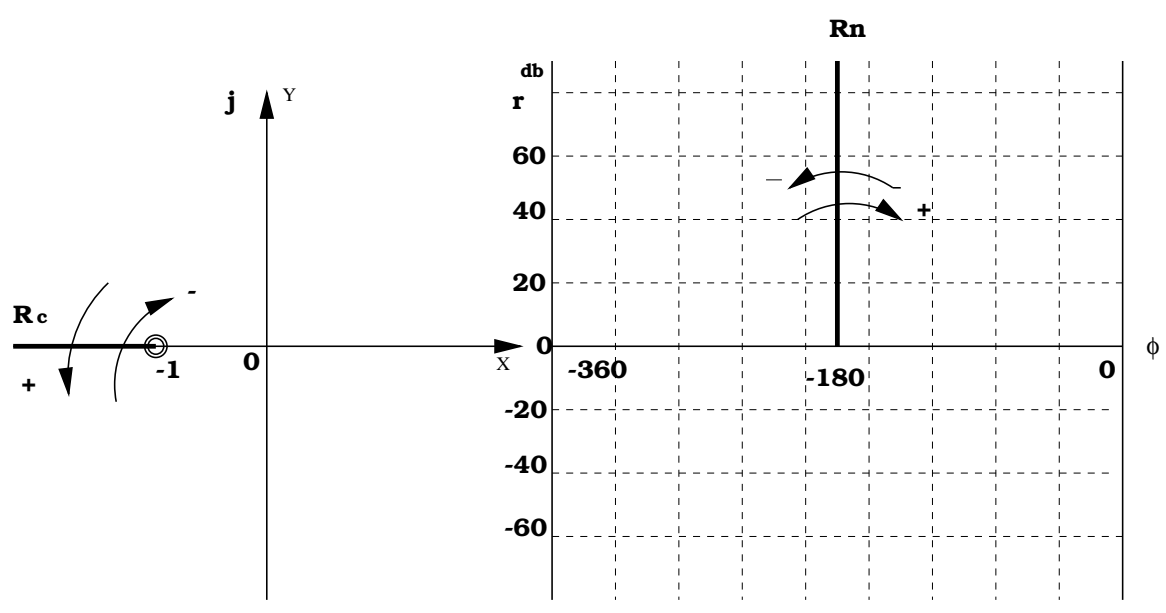

Figure 9: The Definition of Positive and Negative Crossings 

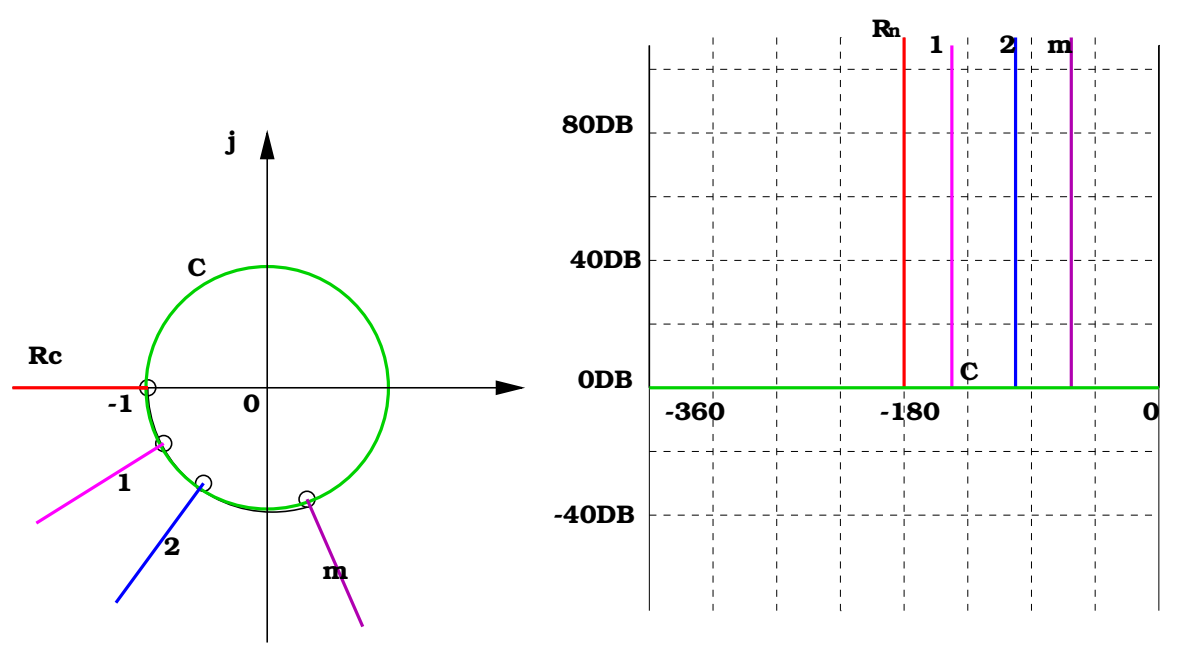

Figure 10: Stability lines on Nyquist plot and Nichols chart for the new nominal plant 


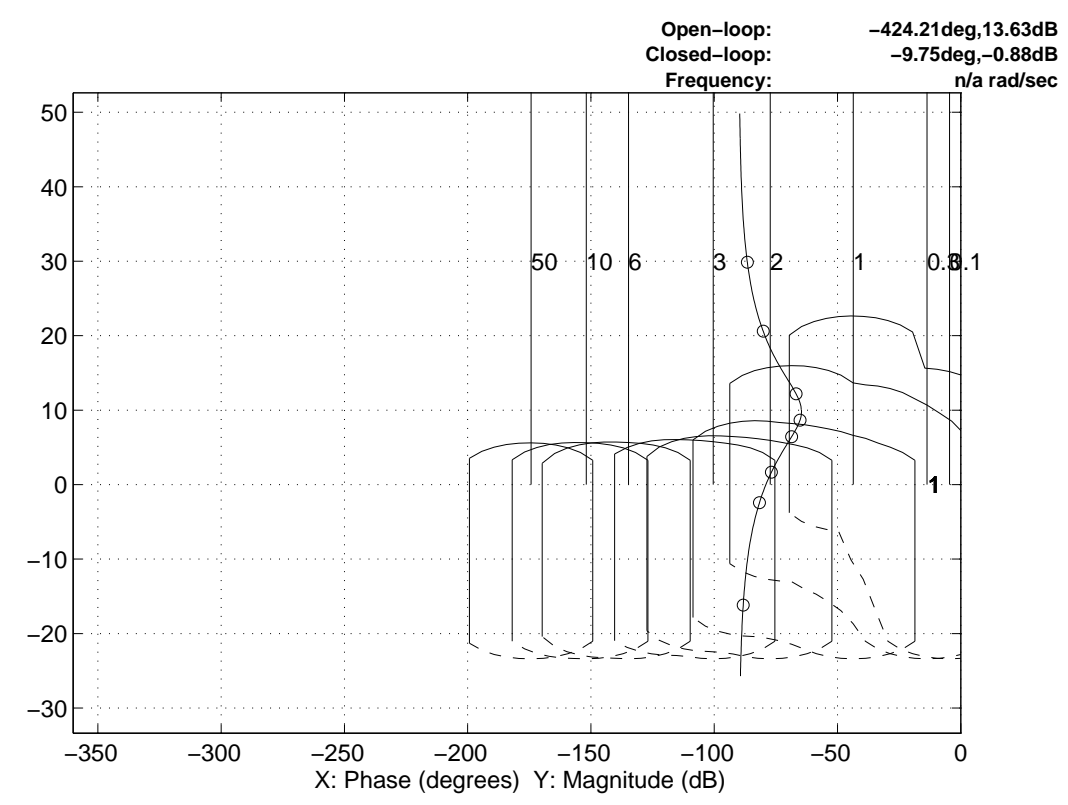

Figure 11: Loop-shaping for Example 2 


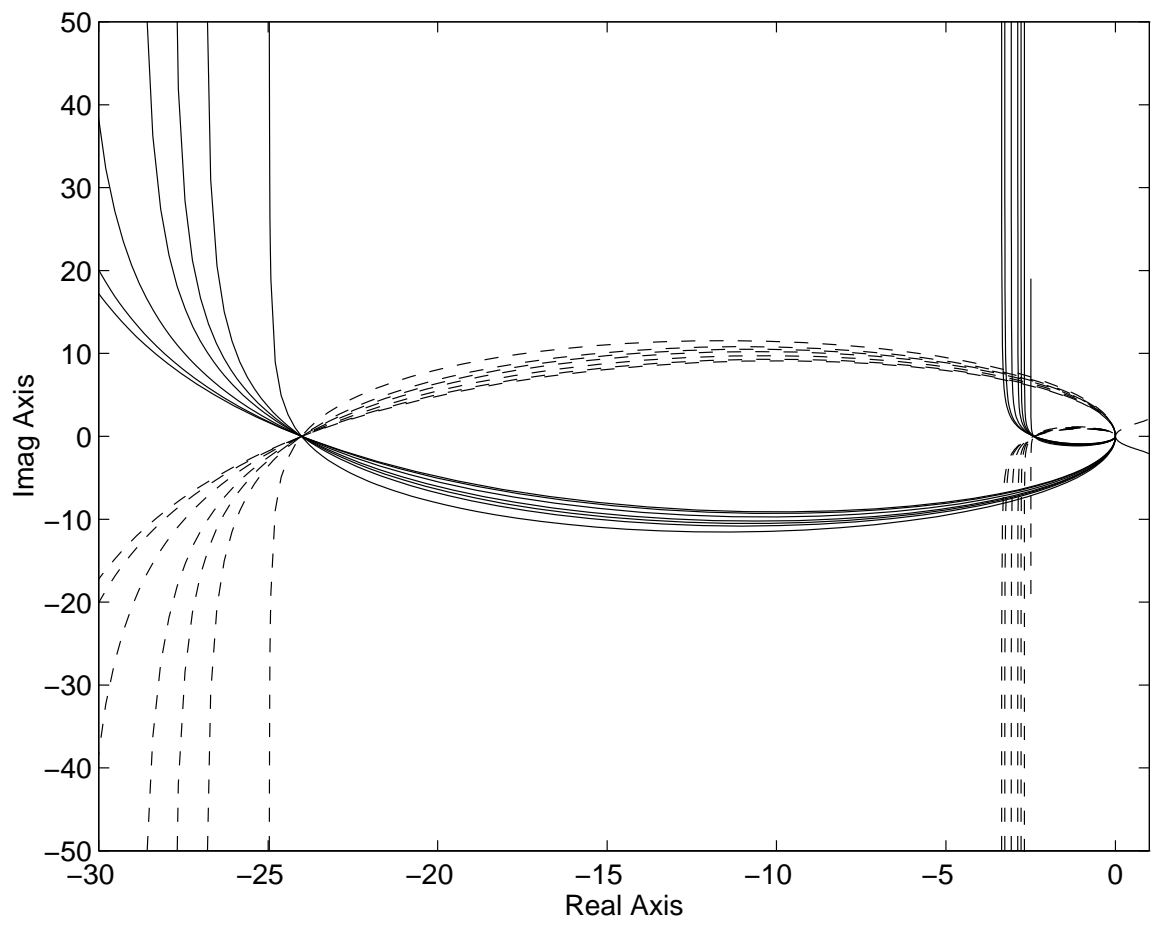

Figure 12: Nyquist plots under the new controller for Example 2 